\title{
Status of CDF Silicon Tracking
}

\author{
S. Nahn ${ }^{\text {a }}$ for the CDF Collaboration \\ aYle University Physics Department \\ PO Box 208121, New Haven, CT 06520-2181
}

A snapshot of the status of the CDF Run II Silicon Detector dated July 2002 is presented, with a summary of commissioning issues since the start of Run II, current performance of the detector, and the use of the data in both the trigger and offline reconstruction.

\section{The CDF Silicon Detector in Run II}

Coincident with the Tevatron Run II upgrade for higher $\sqrt{s}$ and increased luminosity, CDF replaced the Run I silicon detector with an entirely new detector with nearly double the coverage of both the luminous region and pseudorapidity $(\eta)$, the ability to do three dimensional tracking, and enhanced impact parameter resolution for better $\mathrm{B}$ tagging [1,2]. The 8 layer, 704 ladder, 722432 channel detector that resulted is comprised of three subdetectors: SVXII, ISL, and L00 ("Layer Zero Zero"). SVXII is 360 doublesided ladders in a layout of six $15 \mathrm{~cm}$ axial sections $\times$ twelve $30^{\circ} \phi$ slices $\times$ five radial layers between 2.5 and $10.6 \mathrm{~cm}$. ISL covers the area between SVXII and the CDF wire tracker (COT), with 296 double-sided ladders at radii of 20 or 28 $\mathrm{cm}$, in total $1.9 \mathrm{~m}$ long, providing silicon hits out to $|\eta| \leq 2$. L00 is a single-sided layer of 48 ladders mounted directly on the beampipe, $1.5 \mathrm{~cm}$ from the beamline, which enhances the impact parameter resolution. The $r-\phi$ and $r-z$ views of the detector are shown in Figure 1.

The three subdetectors share the same readout system, starting with the SVX3D chip, a custom designed ASIC with a 128 channel $\times 46$ capacitor analog storage ring, which makes it possible to acquire data in a "deadtimeless" fashion, integrating charge on one capacitor while reading out another. The SVX3D chip also features common mode noise suppression, sparsification, and other settings used to optimize the charge collection. A "wedge" of up to 5 ladders are serviced by one
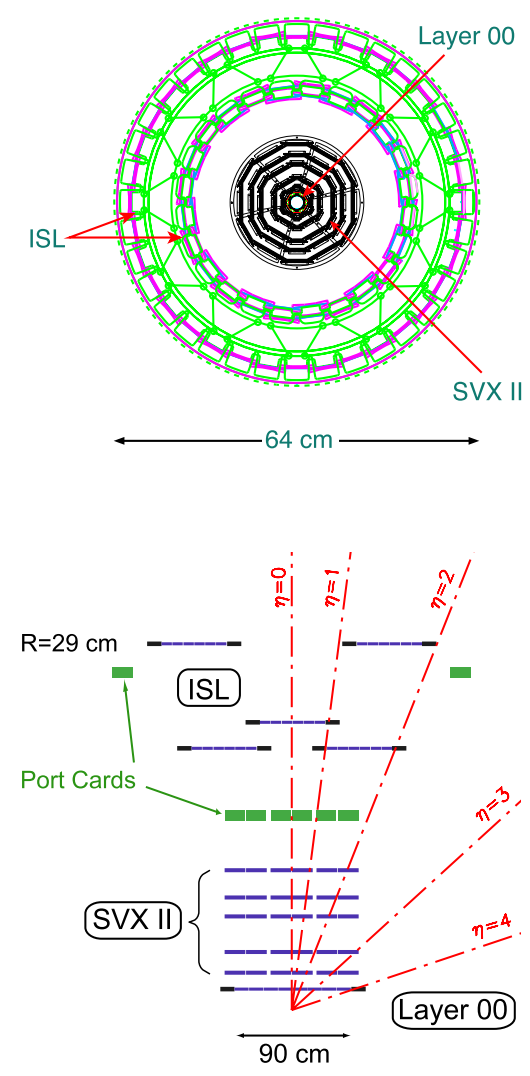

Figure 1. $r-\phi$ and $r-z$ cartoons of the Silicon detector, showing all sub-components. Note the compression of the $z$ scale. 
"portcard" mounted near the detector which provides a $1 \rightarrow 5$ fanout of electrical control signals and converts the data from electrical to optical for long distance transmission, as well as bringing in the analog, digital, and bias voltages to the chips and ladders. Behind this is a VME based data acquisition system which controls the entire detector and presents data to the Level 2 and Level 3 trigger systems. All detectors are incorporated in the same power distribution, cooling, interlock, and radiation protection systems.

\section{Silicon Commissioning Experience}

Commissioning commenced with the onset of Run II in early 2001. Since then, progress has been steady in getting the detector to a robust operating point, but seriously hampered by a series of problems, from which lessons for future detectors can be drawn. The first serious problem was an issue with a vendor, overcome with relentless pursuit. The full detector was not outfitted with power supplies until late October, forcing "supply juggling" to ensure functionality and cabling during installation. Furthermore, the supply interlock mechanisms failed, such that the detector could be powered without cooling. This danger necessitated the shutdown of all Silicon operation until the problem was solved. The second set of problems arose from a lack of testing under realistic conditions. This manifested itself in a variety of ways, most notably the discovery of cooling lines blocked by epoxy in ISL, which have since been bored out with a laser, but also the presence of excess noise in L00, and mismatches in optical power of receivers and transmitters, both of which required much effort to work around. The solutions were developed as soon as the issues were were exposed by the true operating conditions, but could have been seen before. In contrast, the last set of impediments were beam-induced damage to SVX3D chips, and ladder and VME power supplies, the latter of which caused large sections of the detector to be turned off. The diagnosis of the chip damage occupied over a month during which the detector was shut off while scenarios were explored on the bench to understand what was damaged, since the damaged parts are inac- cessible. Considerable delay may have been eliminated had the failure modes been well catagorized before installation. Having all pieces available, testing under operational conditions, and understanding in detail all failure modes are the important lessons to take away from the silicon commissioning experience.

\section{Performance of the Silicon}

Despite the litany of problems described in the previous section, by July 2002 over $90 \%$ of the silicon detector was powered, and more than $80 \%$ reading out quality data. For any ladder type and chip setting on average $\frac{\text { Signal }}{\text { Noise }} \geq 10$, with operating conditions set at 11-15 depending on ladder type. Figure 3 shows a histogram of a typical ladder's pedestal and signal ADC counts, exhibiting clear seperation between the guassian noise and Landau distribution for the signal. With high sig-

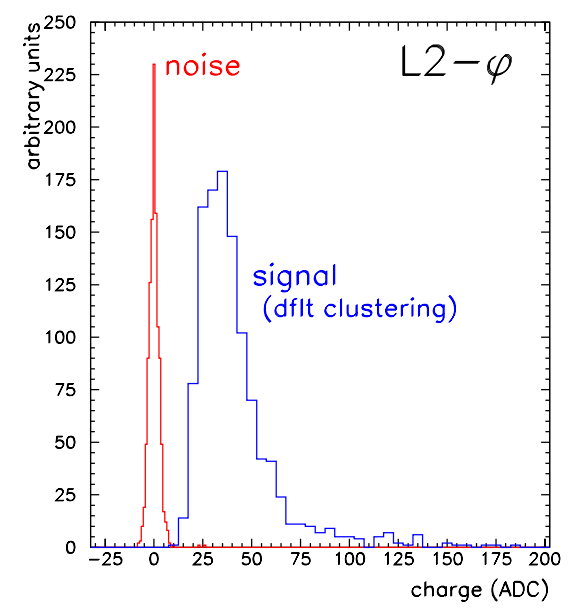

Figure 2. Pedestal and signal for a SVXII L2 $\phi$ side ladder

nal to noise, the single hit efficiencies can be kept $\geq 99 \%$, maintaining high signal efficiency without prohibitive data volume. With tracks in the data both the global and relative alignment continue to improve. In addition, the cluster resolution of 
the detector is as expected, with for example an $11 \mu \mathrm{m}$ intrinsic resolution on 2 strip clusters for the $r-\phi$ measurement, as shown in Figure 3. In general, the raw silicon detector performance

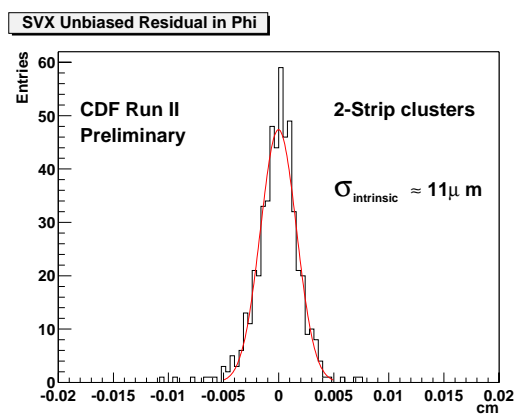

Figure 3. Two-Strip $\phi$ cluster resolution $\sim 11 \mu \mathrm{m}$

shows promise for physics in Run II.

\section{Tracking Applications}

While performance benchmarks are necessary for validation, the real test of the detector is whether one can perform measurements. In addition to traditional offline tracking, the silicon detector is also utilized in the Level 2 trigger every $\sim 50 \mu s$, where tracking information from the COT is combined with silicon hits and high impact parameter tracks are then used to trigger on heavy flavor physics. This allows CDF to trigger on lepton-less B decays, which both relieves the penalty of the small B leptonic branching fraction and enhances the fraction of fully reconstructed $\mathrm{B}$ decays in the data. The details and results of this application were presented to the conference and can be found in [3]. Of course, the detector is also used in offline tracking, and there are results from Run II both in "calibration" channels such as the $\mathrm{J} / \psi$ mass (Figure 4 ) and $\mathrm{B}$ physics channels, as presented in [4]. The conclusion is that this detector is useful for collecting and analyzing interesting physics data.

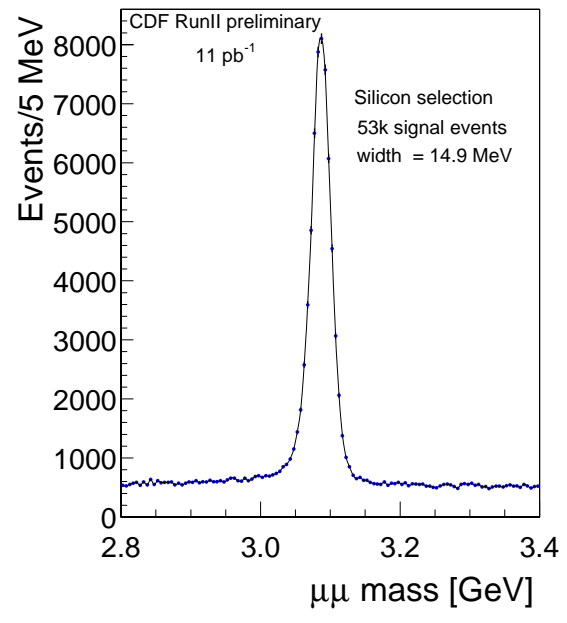

Figure 4. Calibration using the $\mathbf{J} / \psi$ mass

\section{Conclusion}

With the first $\sim 35 p b^{-1}$ of Run II data, the new CDF silicon detector has been commissioned, with some setbacks, and is currently taking data of a quality useful for competitive measurements. The detector will continue to improve as subtle problems are addressed, until the end of its useful lifetime, when the Run IIb detector will be installed [5].

\section{REFERENCES}

1. CDF Collaboration, "The CDF II Detector Technical Design Report", FERMILAB-Pub96/390-E.

2. CDF II Collaboration, "Proposal for Enhancement of the CDF II Detector: An Inner Silicon Layer and a Time of Flight Detector" FERMILAB-Proposal-909

3. A. Cerri, these proceedings

4. F. Bedeschi, these proceedings

5. M. Kruse, these proceedings 